\title{
Reduced Differential Transform Method to Study the Mathematical Model of Tumor Invasion and Metastasis
}

\author{
Khushbu D. Patel ${ }^{1}$, D. C. Joshi ${ }^{2}$ \\ Department of Mathematics \\ Government Engineering College, \\ Valsad-396001, Gujarat, Indial \\ Department of Mathematics, \\ Veer Narmad South Gujarat University, \\ Surat-7, Gujarat, India ${ }^{2}$ \\ Email:kvpatel246@gmail.com ${ }^{1}$,dilip_joshi2002@yahoo.co.in ${ }^{2}$
}

\begin{abstract}
In this paper we present Non-linear mathematical model of tumor Invasion and Metastasis. Model focuses on three key variables involved in invasion process, namely, tumor cells, host tissue (Extracellular matrix) and matrix degradative enzymes associated with the tumor cells. The model gives system of non-linear partial differential equations. The Reduced Differential Transform Method is applied to obtain the approximate solution of this model. The study shows the ability of the method for solving nonlinear partial differential equations. It is observed that the method does not required linearization and weak nonlinearity assumptions and is very simple in computation.
\end{abstract}

Keywords- Tumor cells, Extracellular matrix, matrix degradative enzymes, reduced differential Transform Method

\section{INTRODUCTION}

Cancer invasion is a cell and tissue-driven process that includes physical, cellular, and molecular determinants to adapt and react throughout its progression (Friedl P. et al., 2011). Most of the clinical tumor patients die from tumor invasion and metastasis. Tumor development is a very complex multistep process involving many intracellular and extracellular phenomena which are strongly nonlinear and time varying (d'Onofrio, A., 2006 \& 2008; Bellomo N. et al., 2006). An important role in the process of cancer invasion is performed by matrix degradative enzymes (MDEs) such as metalloproteases (MMPs). They are produced by tumor cells and digest the ECM, which enables the migration of cancer cells through the tissue (Liotta, L. A., 1983; Stevenson et al., 1993).

Within the last three decades a number of mathematical models for tumor growth have been developed as part of the quest to understand tumor growth dynamics. Gatenby and Gawlinski present one of the first models of tumor invasion in the papers (Gatenby, R. A. et al., 1995 \& 1996) considers the competition between healthy host cells and modified (tumour) cells and proposes and analyses several models formulated in terms of ordinary differential equations. Gatenby and Gawlinski (Gatenby, R. A. et al., 1996) present a reaction-diffusion model for the

investigation of the role of the alteration of the microenvironmental acidity induced by cancer cells for their invasion into the organism. In this paper, we study the continuum models of avascular tumor growth investigated by (Chaplain et al.1996, 2000 \& 2006). Chaplain used numerical solution (finite difference method) to solve the above problem. A variety of methods, exact, approximate, and purely numerical are available for the solution of this type of problems such as Adomian decomposition method, Homotopy perturbation method and Differential Transform method.

The reduced differential transform method (RDTM) was first proposed by the Turkish mathematician Keskin and Oturanc in the year 2009 to solve linear and non-linear ordinary as well as partial differential equations (Keskin, Y. et al., 2011). This method has received much attention since then and applied to solve a wide variety of problems. This reduced differential transform method is introduced mainly to overcome the demerits of complex calculation of the usual differential transform method. Then a number of authors like (Keskin, Y. et al., 2009 \& 2010), (Cenesiz, et.al. 2010), (Taha B. A., 2011), (Taha and Wahab, 2012) solved many equations using this method. This paper is presented to solve tumor invasion and metastasis model by RDTM

\section{PROBLEM FORMULATION}

In our model, we focus on three key variables implicated in the Tumor invasion process, namely, tumor cells, host tissue (extracellular matrix) and matrix- degradative enzymes associated with the 


\section{Available online at www.ijrat.org}

tumor cell. By definition, haptotaxis is the directed migratory response of cells to gradients of fixed or bound chemicals. Also, haptotaxis is a major component of directed movement in tumour cell invasion. Indeed, there has been much recent effort to characterise such directed movement (Klominek, J. et al., 1993; Lawrence J.A. et al., 1996). We therefore refer to this directed movement of tumor cells in our model as haptotaxis. To incorporate this directed movement of tumor cells in our model, we take the haptotactic flux to be

$$
J_{\text {hapto }}=\chi n \Delta f
$$

Where $\chi$ is the (positive constant) haptotactic coefficient.

And to describe the random motility of the tumor cells we assume a flux of the form

$$
J_{\text {random }}=-D(f, m) \nabla n
$$

Where $D(f, m)$ may be a constant or a function of either the MDE or ECM concentration.

In our partial differential equation model, we do not consider any proliferation of tumor cells. We focus entirely on the cell-matrix interactions and how these interactions affect tumor cell migration. The conservation equation for the tumor cell density $n$ (from Eq. (1) and (2)) is therefore given by

$$
\frac{\partial n}{\partial t}+\nabla \cdot\left(J_{\text {random }}+J_{\text {hapto }}\right)=0
$$

Hence, the partial differential equation governing tumor cell motion (in the absence of cell proliferation) is,

$$
\frac{\partial n}{\partial t}=\nabla \cdot(D(f, m) \nabla n)-\chi \nabla \cdot(n \nabla f)
$$

In our model we choose $D(f, m)=D_{n}$, a constant, the tumor cell random motility coefficient.

The ECM contains many macro- molecules, including fibronectin, laminin and collagen, which can be degraded by MDEs (Chambers, A. F. et al., 1997). We assume that the MDEs degrade ECM upon contact and hence the degradation process is modeled by

$$
\frac{\partial f}{\partial t}=-\delta m f
$$

Where $\delta$ is a positive constant.

Active MDEs are produced (or activated) by the tumor cells, diffuse throughout the tissue and undergo some form of decay (either passive or active). Therefore, the governing equation of MDE concentration is given by:

$$
\frac{\partial m}{\partial t}=D_{m} \nabla^{2} m+g(n, m)-h(n, m, f)
$$

Where $D_{m}$ is a positive constant, the MDE diffusion coefficient, $g=\mu n$ (MDE production by the tumor cells) and $h=\lambda m$ (natural decay), as for simplicity we assume that there is a linear relationship between the density of tumor cells and the level of active MDEs in the surrounding tissues. Hence the complete system describing the interactions of the tumor cells (denoted by $n$ ), extra cellular matrix (ECM, denoted by $f$ ), and matrix degrading enzymes (MDE, denoted by $m$ ) is given by (Gatenby, R. A. et al, 1996)

$$
\frac{\partial n}{\partial t}=D_{n} \nabla^{2} n-\chi \nabla \cdot(n \nabla f)
$$

$$
\frac{\partial f}{\partial t}=-\delta m f
$$

$$
\frac{\partial m}{\partial t}=D_{m} \nabla^{2} m+\mu n-\lambda m
$$

Where $D_{n}$ is the tumor cell random motility coefficient, $D_{m}$ is the MDE diffusion coefficient, $\chi$ is the haptotactic coefficient, and $\lambda, \mu, \delta$ are the positive constants. Non-dimensionalise of Eq. (7), (8), (9) by setting

$$
\bar{n}=\frac{n}{n_{0}}, \bar{f}=\frac{f}{f_{0}}, \bar{m}=\frac{m}{m_{0}}, \bar{x}=\frac{x}{L}, \bar{t}=\frac{t}{\tau}
$$

Where $n_{0}$ is the tumor cell density, $f_{0}$ is the ECM density, $m_{0}$ is the MDE concentration, $L$ is the length scale, and $\tau$ is the time ( $\tau=\frac{L^{2}}{D}$, where $D$ is a reference chemical diffusion coefficient). By dropping the tildes for notational convenience, we obtain the scaled system of equations:

$$
\frac{\partial n}{\partial t}=d_{n} \nabla^{2} n-\gamma \nabla \cdot(n \nabla f)
$$

$\frac{\partial f}{\partial t}=-n m f$

$\frac{\partial m}{\partial t}=d_{m} \nabla^{2} m+\omega n-\beta m$

Where $d_{n}=D_{n} / D, \quad \gamma=\chi f_{0} / D, \quad \eta=\tau m_{0} \delta$, $d_{m}=D_{m} / D, \omega=\tau \mu_{n_{0}} / m_{0}$ and $\beta=\tau \lambda$ 


\section{Available online at www.ijrat.org}

We assume that initially there is a nodule of cells already present and the tumor is centered on $x=0$ with $n$ having density distribution.

$$
n(x, 0)=\exp \left(-\frac{x^{2}}{\in}\right)
$$

Also, we suppose that the tumor has already degraded some of its surrounding tissue and so initially profile of ECM to be,

$$
f(x, 0)=1-0.5 \exp \left(-\frac{x^{2}}{\in}\right)
$$

Finally, we assume that the initial MDE concentration profile is proportional to the $\mathrm{n}(\mathrm{x}, 0)$ and then we assume,

$$
m(x, 0)=0.5 \exp \left(-\frac{x^{2}}{\epsilon}\right)
$$

Where $\epsilon$ is a positive constant. We assume that there is no-flux of tumor cells or MDE across the boundary of the domain, namely $x=0$ and $x=1$. These boundary conditions are represented by the following equations,

$$
-D_{n} \frac{\partial n}{\partial x}+n \chi \frac{\partial f}{\partial x}=0 \quad \text { And } \frac{\partial m}{\partial x}=0, \text { at } x=0,1
$$

\section{REDUCED DIFFERENTIAL TRANSFORM METHOD (RDTM)}

The basic definition of the reduced differential Transform method is as follows:

Definition-1: If the function $u(x, t)$ is analytic and differentiable continuously with respect to time $t$ and space $x$ in the domain of interest, then

$U(k)=\frac{1}{k !}\left[\frac{\partial^{k}}{\partial x^{k}} u(x, t)\right]$ at $t=0$

Where the t-dimensional spectrum function $U(k)$ is the transformed function of $u(x, t)$.Here the lower case function $u(x, t)$ represents the original function while the upper case function $U(k)$ stands for the transformed function.

Definition 2: The inverse reduced differential transform of $U(k)$ is defined as follows:

$$
u(x, t)=\sum_{k=0}^{\infty} U(k) t^{k}
$$

Thus combining Eq. (17) and (18), we can express the solution as follows:

$u(x, t)=\sum_{k=0}^{\infty}\left(\frac{1}{k !}\left[\frac{\partial^{k}}{\partial x^{k}} u(x, t)\right]\right.$ at $\left.t=0\right) t^{k}$

The basic concept of reduced differential transform method mainly comes from the power series

\begin{tabular}{|c|c|}
\hline Original Function & Transformed function \\
\hline$u(x, t)$ & $\begin{array}{l}U(k)=\frac{1}{k !}\left[\frac{\partial^{k}}{\partial x^{k}} u(x, t)\right] \quad \text { at } \\
t=0\end{array}$ \\
\hline$w(x, t)=u(x, t) \pm v(x, t)$ & $W(k)=U(k) \pm V(k)$ \\
\hline$w(x, t)=c u(x, t)$ & $\begin{array}{l}W(k)=c U(k), \text { where } \mathrm{c} \text { is } \\
\text { constant }\end{array}$ \\
\hline$w(x, t)=x^{m} t^{n}$ & $\begin{array}{l}W(k)=x^{m} \delta(k-n) \\
\text { where } \delta(\mathrm{k}-\mathrm{n})=\left\{\begin{array}{l}1, k=n \\
0, k \neq n\end{array}\right.\end{array}$ \\
\hline$w(x, t)=x^{m} t^{n} u(x, t)$ & $W(k)=x^{m} U(k-n)$ \\
\hline$w(x, t)=u(x, t) v(x, t)$ & $W(k)=\sum_{r=0}^{k} U(r) V(k-r)=\sum_{r=0}^{k} V(r) U(k-$ \\
\hline$w(x, t)=\frac{\partial^{r}}{\partial t^{r}} u(x, t)$ & $W(k)=\frac{(k+r) !}{k !} U(k+r)$ \\
\hline$w(x, t)=\frac{\partial}{\partial x} u(x, t)$ & $W(k)=\frac{\partial}{\partial x} U(k)$ \\
\hline$w(x, t)=\frac{\partial^{2}}{\partial x^{2}} u(x, t)$ & $W(k)=\frac{\partial^{2}}{\partial x^{2}} U(k)$ \\
\hline
\end{tabular}
expansion. Few fundamental mathematical operations performed by this reduced differential method are listed below:

Table 1. Some Transformed functions

\section{SOLUTION BY RDTM:}

Applying RDTM to Eq. (11), (12) \& (13) we have following equations:

$(k+1) N(k+1)=d_{n} \frac{\partial^{2}}{\partial x^{2}} N(k)-$

$\gamma\left[\sum_{r=0}^{k} \frac{\partial}{\partial x} N(k-r) \frac{\partial}{\partial x} F(r)+\sum_{r=0}^{k} \frac{\partial^{2}}{\partial x^{2}} F(r)\right]$ 
International Journal of Research in Advent Technology, Vol.7, No.2, February 2019

E-ISSN: 2321-9637

Available online at www.ijrat.org

$(k+1) F(k+1)=-\eta \sum_{r=0}^{k} M(k-r) F(r)$

$(k+1) M(k+1)=d_{m} \frac{\partial^{2}}{\partial x^{2}} M(k)+\omega N(k)$

$-\beta M(k)$

Also applying RDTM to the Initial condition Eq. (14),

(15) \& (16), we have,

$$
\begin{aligned}
& N(0)=\exp \left(-\frac{x^{2}}{\epsilon}\right) \quad F(0)=1-0.5 \exp \left(-\frac{x^{2}}{\epsilon}\right) \\
& M(0)=0.5 \exp \left(-\frac{x^{2}}{\epsilon}\right)
\end{aligned}
$$

Now taking $k=0$ in Eq. (20), (21), (22) and using Eq. (23), we have,

$$
\begin{aligned}
& N(1)=-\frac{e^{\frac{-x^{2}}{\epsilon}}}{\epsilon}\left[2 d_{n}\left(1-\frac{2 x^{2}}{\epsilon}\right)+\gamma e^{\frac{-x^{2}}{\epsilon}}\left(1-\frac{4 x^{2}}{\epsilon}\right)\right] \\
& F(1)=-\frac{\eta e^{\frac{-x^{2}}{\epsilon}}}{2}\left(1-\frac{e^{\frac{-x^{2}}{\epsilon}}}{2}\right) \\
& M(1)=-e^{\frac{-x^{2}}{\epsilon}}\left[\frac{d_{m}}{\epsilon}\left(1-\frac{2 x^{2}}{\epsilon}\right)-\omega+\frac{\beta}{2}\right]
\end{aligned}
$$

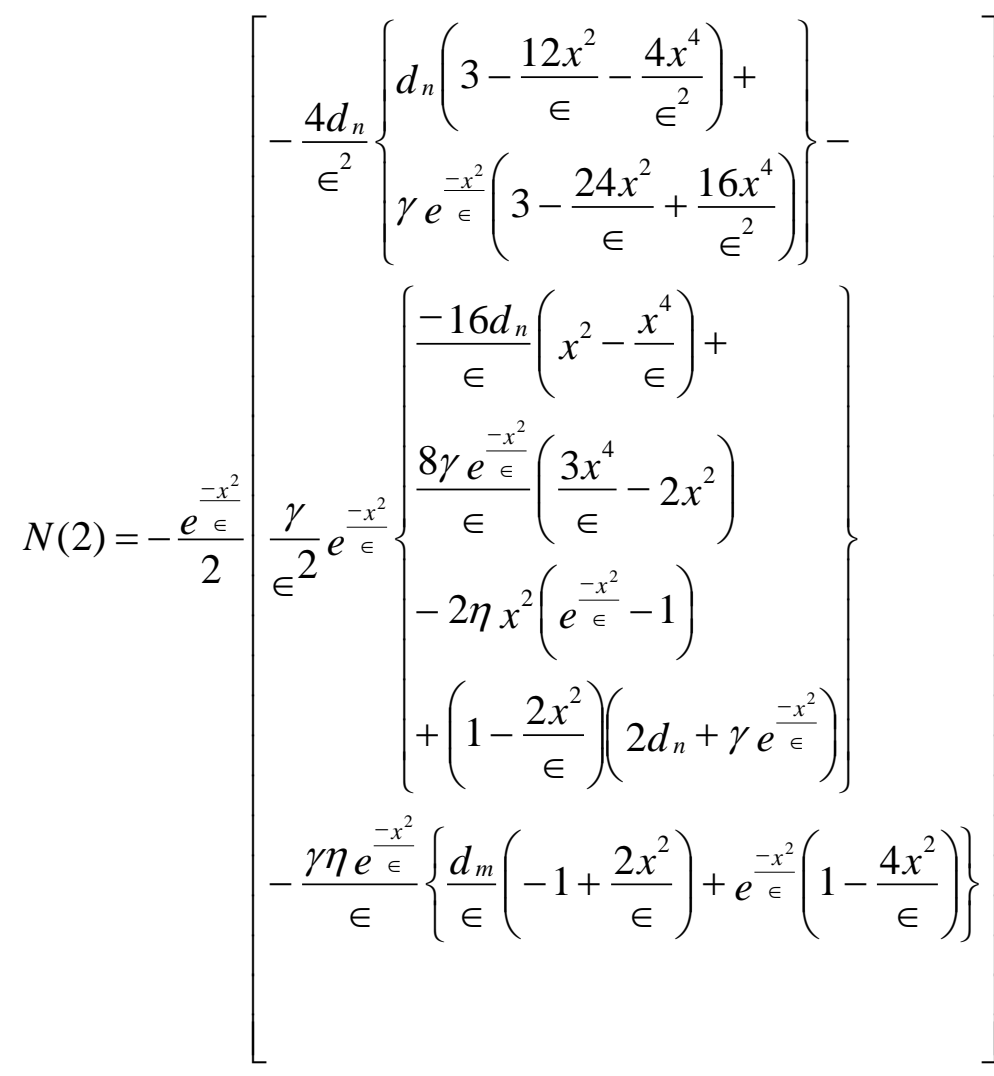

Again for $k=1$ in recurrence relation, we have,

$$
\begin{aligned}
& F(2)=\frac{\eta}{2} e^{\frac{-x^{2}}{\epsilon}}\left(1-\frac{e^{\frac{-x^{2}}{\epsilon}}}{2}\right)\left[\frac{d_{m}}{\epsilon}\left(1-\frac{2 x^{2}}{\epsilon}\right)-\omega+\frac{\beta}{2}+\frac{\eta e^{\frac{-x^{2}}{\epsilon}}}{4}\right] \\
& d_{m}\left\{\begin{array}{l}
\frac{2 d_{m}}{\epsilon^{2}}\left(-3+\frac{12 x^{2}}{\epsilon}-\frac{4 x^{4}}{\epsilon^{2}}\right)+ \\
\frac{1}{\epsilon}\left(1-\frac{2 x^{2}}{\epsilon}\right)(2 \omega-\beta)
\end{array}\right\} \\
& \left.M(2)=-\frac{e^{\frac{-x^{2}}{\epsilon}}}{2}\right)+\omega\left\{\frac{2 d_{n}}{\epsilon}\left(1-\frac{2 x^{2}}{\epsilon}\right)+\frac{\gamma e^{\frac{-x^{2}}{\epsilon}}}{\epsilon}\left(1-\frac{4 x^{2}}{\epsilon}\right)\right\} \\
& -\beta\left\{\frac{d_{m}}{\in}\left(1-\frac{2 x^{2}}{\epsilon}\right)-\omega+\frac{\beta}{2}\right\}
\end{aligned}
$$

And so on

Finally, the inverse reduced differential transform of $N(k), F(k), M(k)$ are obtained from following relations 


$$
n(x, t)=\sum_{k=0}^{\infty} N(k) t^{k}, \quad f(x, t)=\sum_{k=0}^{\infty} F(k) t^{k}, \quad m(x, t)=\sum_{k=0}^{\infty} M(k) t^{k}
$$

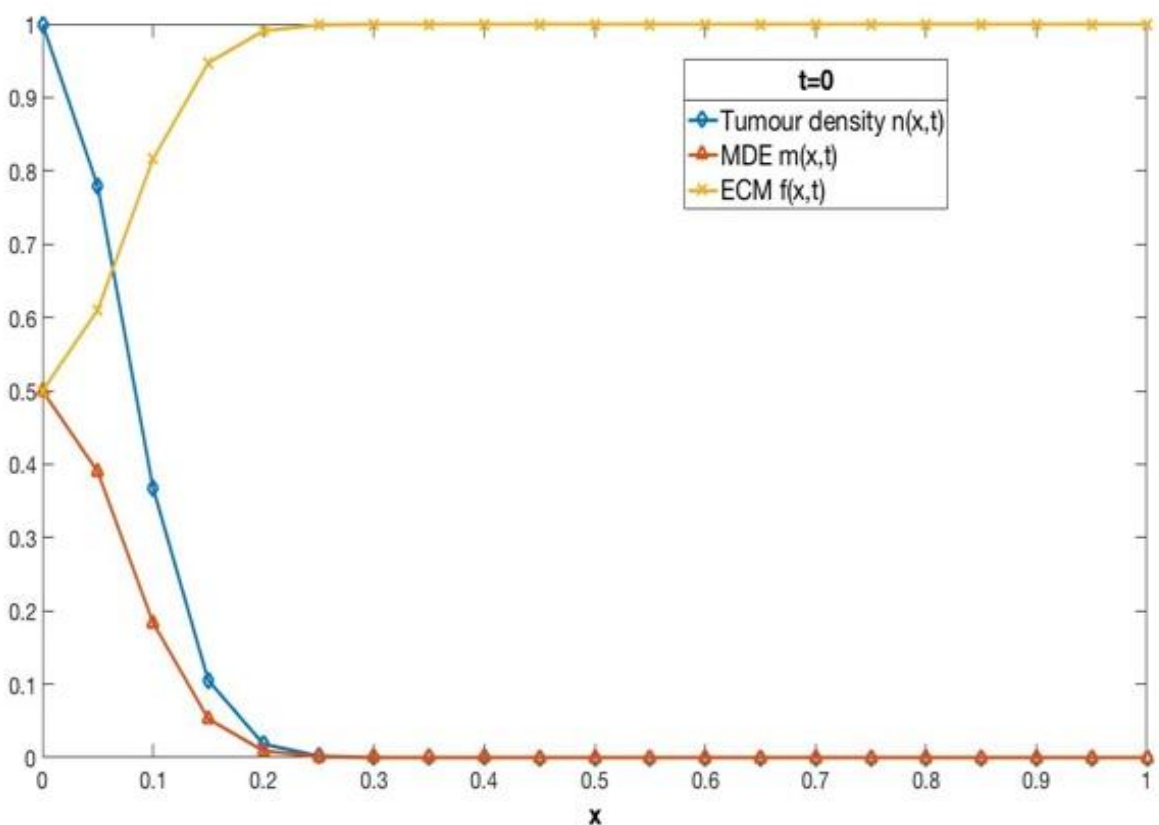

Figure 1 One-dimensional RDTM solution of system of Eq. (11)-(13) with constant tumor cell diffusion showing the cell diffusion showing the cell density, MDE concentration and ECM density for $t=0$,

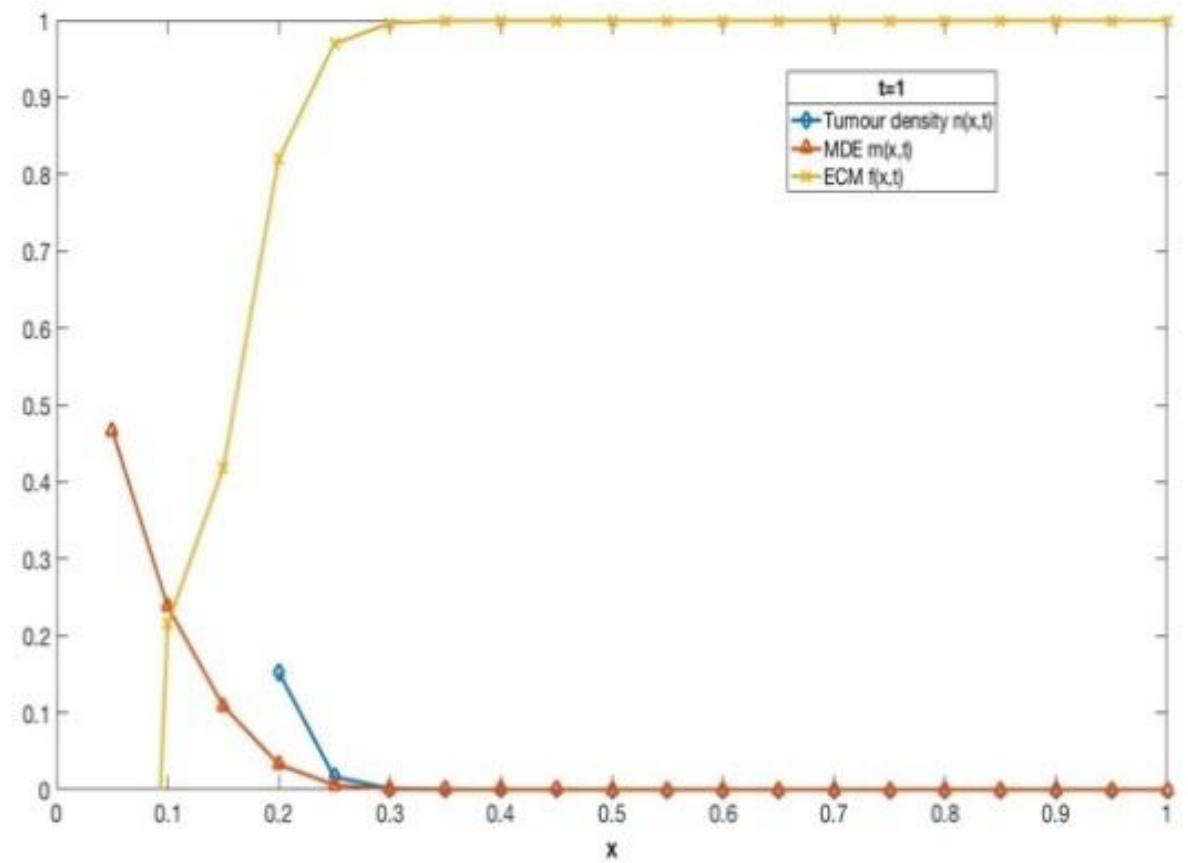

Figure 2 One-dimensional RDTM solution of system of Eq. (11)-(13) with constant tumor cell diffusion showing the cell diffusion showing the cell density, MDE concentration and ECM density for $t=1$, 
Available online at www.ijrat.org

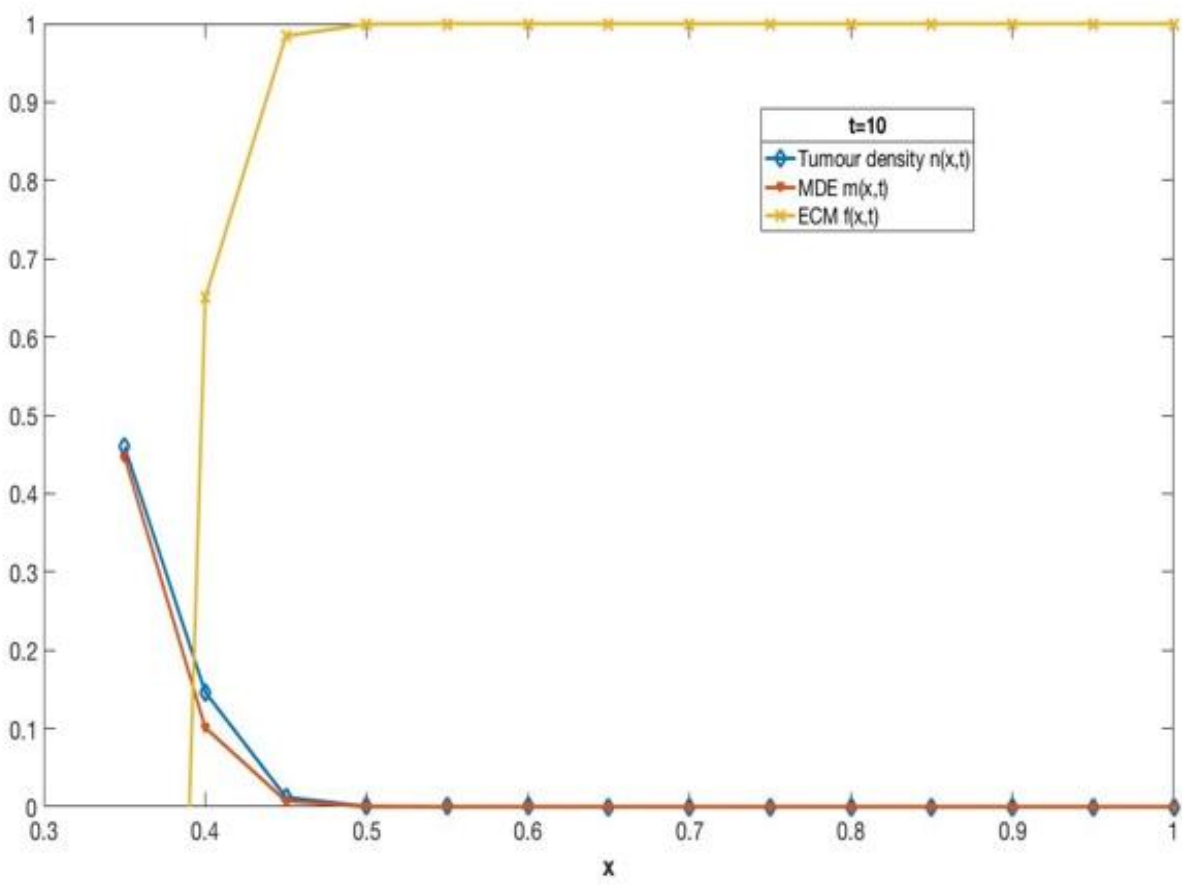

Figure 3 One-dimensional RDTM solution of system of Eq. (11)-(13) with constant tumor cell diffusion showing the cell diffusion showing the cell density, MDE concentration and ECM density for $t=10$,

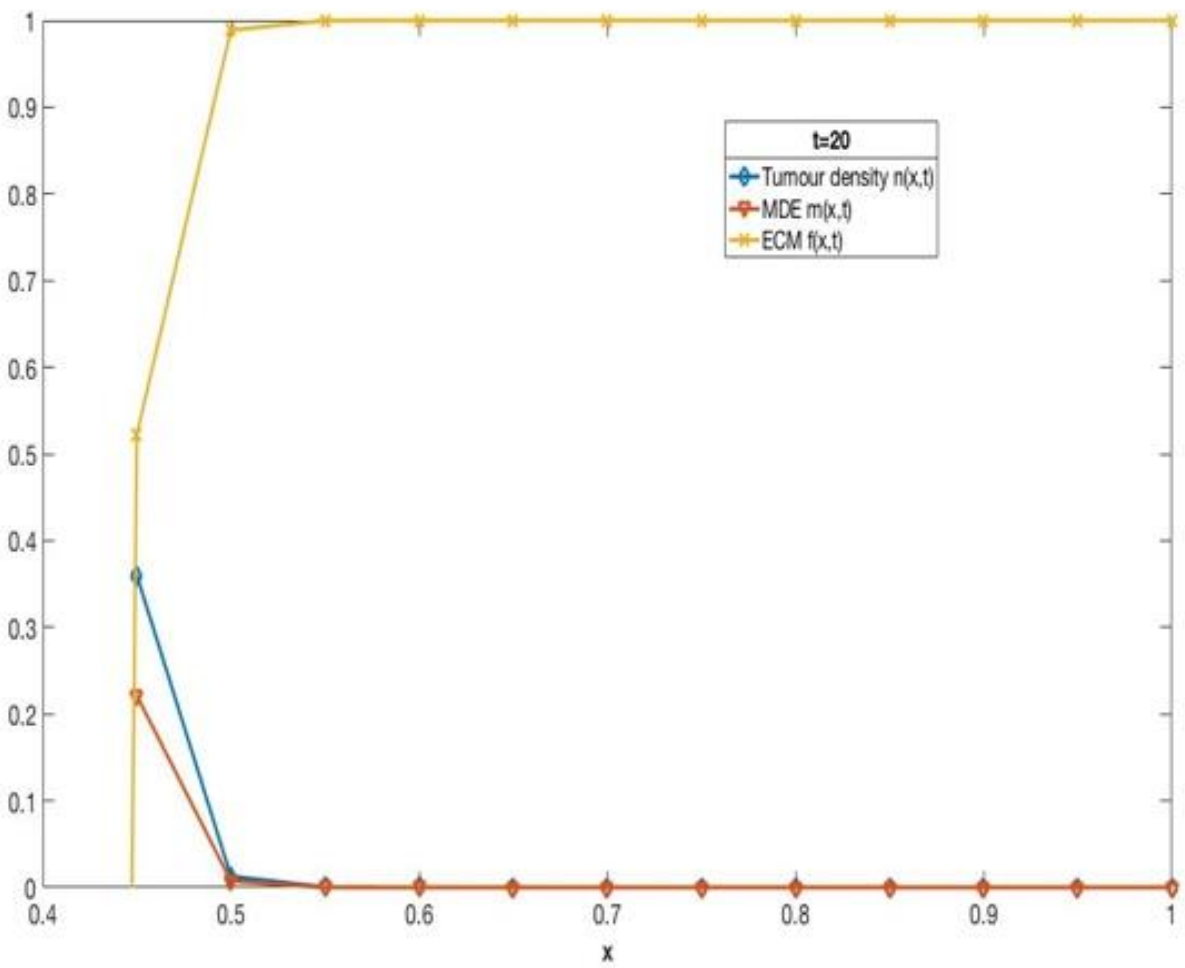

Figure 4 One-dimensional RDTM solution of system of Eq. (11)-(13) with constant tumor cell diffusion showing the cell diffusion showing the cell density, MDE concentration and ECM density for $t=20$, 


\section{Available online at $w w w . i j r a t . o r g$}

\section{RESULT AND CONCLUSIONS}

Figure 1-4 show four snapshots in time of the tumor cell density, ECM density, and MDE concentration. Initially, by $t=1$ cancer cells have migrated a small distance into the domain. By $t=10$ cancer cells have migrated almost half way through the domain. And as time evolves by $t=20$ cancer cells continue to migrate to regions where high ECM densities are situated. Also, the ECM profile shows clearly the degradation by the MDEs. As the MDEs degrade the ECM, the tumor cells invade via combination of diffusion and haptotaxis. The tumor density distribution shows a small cluster of cells built up at the leading edge of the tumor due to haptotactic migration. As time evolves, this cluster of cells migrates further from the tumor main body and continues to invade the ECM at slower rate.

In this paper, Reduced Differential Transform method is applied for the approximate solution of mathematical model of tumor invasion and metastasises. Results obtained from RDTM are very near to the results obtained from other Numerical methods like Adomian Decomposition Method and Homotopy Perturbation Method (Mahiddin, N. et al., 2014). As RDTM is very simple in computation compare to the other method, thus it is very effective and convenient. We suggest applying this method to solve other Biological problems.

\section{REFERENCES}

[1] Anderson, A. R., Chaplain, M. A., Newman, E. L., Steele, R. J., \& Thompson, A. M. Mathematical modelling of tumor invasion and metastasis. Computational and mathematical methods in medicine, 2(2), 129-154,2000.

[2] Bellomo, N., \& Bellouquid, A. On the onset of non-linearity for diffusion models of binary mixtures of biological materials by asymptotic analysis. International Journal of Non-Linear Mechanics, 41(2), 281-293, 2006.

[3] Cenesiz, Y., Keskin, Y., \&Kurnaz, A. The Solution of the Nonlinear Dispersive $K(m, n)$ Equations by RDT Method. International Journal of Nonlinear Science, 9(4), 461-467, 2010.

[4] Chambers, A. F., \&Matrisian, L. M. Changing views of the role of matrix metalloproteinases in metastasis. Journal of the National Cancer Institute, 89(17), 1260-1270, 1997.

[5] Chaplain, M. A. J. Avascular growth, angiogenesis and vascular growth in solid tumors: The mathematical modeling of the stages of tumor development. Mathematical and computer modelling, 23(6), 47-87,1996.
[6] Chaplain, M. A., \&Lolas, G. Mathematical modeling of cancer invasion of tissue: dynamic heterogeneity. NHM, 1(3), 399-439, 2006.

[7] d'Onofrio, A. Tumor-immune system interaction: modelling the tumor-stimulated proliferation of effectors and immunotherapy. Mathematical Models and Methods in Applied Sciences, 16(08), 1375-1401,2006.

[8] d'Onofrio, A. Metamodeling tumor-immune system interaction, tumor evasion and immunotherapy. Mathematical and Computer Modeling, 47(5-6), 614-637,2008.

[9] Friedl, P., \& Alexander, S. Cancer invasion and the microenvironment: plasticity and reciprocity. Cell, 147(5), 992-1009,2011.

[10] Gatenby, R. A. Models of tumor-host interaction as competing populations: implications for tumor biology and treatment. Journal of Theoretical Biology, 176(4), 447-455, 1995.

[11] Gatenby, R. A., \&Gawlinski, E. T. A reactiondiffusion model of cancer invasion. Cancer research, 56(24), 5745-5753, 1996.

[12] Keskin, Y., \&Oturanc, G. Reduced differential transform method for partial differential equations. International Journal of Nonlinear Sciences and Numerical Simulation, 10(6), 741750, 2009.

[13] Keskin, Y., \&Oturanç, G. Application of reduced differential transformation method for solving gas dynamics equation. International Journal of Contemporary Mathematical Sciences, 22(22), 1091-1096, 2010.

[14] Keskin, Y., \&Oturanc, G. Numerical solution of regularized long wave equation by reduced differential transform method. Applied Mathematical Sciences, 4(25), 1221-1231, 2010.

[15] Keskin, Y., Servi, S., \&Oturanç, G. Reduced differential transform method for solving Klein Gordon equations. In Proceedings of the World Congress on Engineering (WCE), 2011.

[16] Klominek, J., Robért, K. H., \& Sundqvist, K. G. Chemotaxis and haptotaxis of human malignant mesothelioma cells: effects of fibronectin, laminin, type IV collagen, and an autocrine motility factor-like substance. Cancer research, 53(18), 4376-4382, 1993.

[17] Lawrence, J. A., \&Steeg, P. S. Mechanisms of tumor invasion and metastasis. World journal of urology, 14(3), 124-130, 1996.

[18] Liotta, L. A. Tumor invasion and the extracellular matrix. Lab invest, 49, 636-649, 1983.

[19] Mahiddin, N., \&Hashim Ali, S. A. Approximate analytical solutions for mathematical model of tumour invasion and metastasis using modified Adomian decomposition and homotopy perturbation methods. Journal of Applied Mathematics, 2014. 
International Journal of Research in Advent Technology, Vol.7, No.2, February 2019

E-ISSN: 2321-9637

Available online at www.ijrat.org

[20] Stetler-Stevenson, W. G., Aznavoorian, S., \& Liotta, L. A. Tumor cell interactions with the extracellular matrix during invasion and metastasis. Annual review of cell biology, 9(1), 541-573, 1993.

[21] Taha, B. A. The use of reduced differential transforms method for solving partial differential equations with variable coefficients. Journal of Basrah Researches (Sciences), 37(4), 2011.

[22] Taha, B. A., \& Abdul-Wahab, R. D. Numerical Solutions of Two-dimensional Burgers' Equations Using Reduced Differential Transform Method. JOURNAL OF SCIENCE, 3(3), 252-264, 2012. 